\title{
NIVEL DE ACTIVIDAD FÍSICA HABITUAL EN ESCOLARES DE 8-9 AÑOS DE ESPAÑA E INDIA
}

\section{LEVEL OF HABITUAL PHYSICAL ACTIVITY IN 8-9 YEARS OLD SCHOOLCHILDREN FROM SPAIN AND INDIA}

\author{
Guillermo Felipe López Sánchez ${ }^{1}$ \\ MD Dilsad Ahmed ${ }^{2}$ \\ Francisco José Borrego Balsalobrel \\ Laura López Sánchez ${ }^{l}$ \\ Arturo Díaz Suárez ${ }^{l}$ \\ Facultad de Ciencias del Deporte, Universidad de Murcia, España ${ }^{l}$ \\ Faculty of Education, Universtiy of Macau, China ${ }^{2}$ \\ gfls@um.es ${ }^{l}$
}

\begin{abstract}
Resumen
El nivel de actividad física de las personas es un tema prioritario a escala internacional. Sobre este tema, el objetivo del presente artículo fue analizar y comparar el nivel de actividad física habitual de escolares de 8-9 años de la región de Murcia, España y el Distrito de Karbi Anglong, Assam en India. Para ello, se administró el cuestionario PACE (Physician-based Assessment and Counseling for Exercise) a un total de 85 escolares (44 de España y 41 de India). Los resultados evidenciaron que la muestra estudiada no realiza suficiente actividad física, según las recomendaciones de la Organización Mundial de la Salud, ya que realizan al menos 60 minutos de actividad física; una media de 3.13 días/semana ( $\mathrm{DE}=1.91)$. Los escolares de 8 años son ligeramente más activos que los de 9 años en ambos países; los niños realizan más actividad física que las niñas en ambos países; los escolares de India realizan más actividad física que los de España. Por tanto, se concluye que el nivel de actividad física es insuficiente, disminuye ligeramente con la edad y es mayor en India.
\end{abstract}

Palabras Claves: Actividad física, Salud, Escolares, Niños, España, India.

\begin{abstract}
The level of physical activity of people is a very important issue internationally. The aim of this study was to analyze and compare the level of habitual physical activity in 8-9 years old schoolchildren from Spain and India. With this purpose, it was administered the questionnaire PACE (Physician-based Assessment and Counseling for Exercise) in a sample of 85 schoolchildren (44 from Spain and 41 from India). The results showed that the sample studied does not do enough physical activity, according to the recommendations of the World Health Organization, as they do at least 60 minutes of physical activity; only an average of 3.13 days/week $(\mathrm{SD}=1.91)$. 8-year-old schoolchildren do more physical activity than 9-year-old schoolchildren in both countries; boys do more physical activity than girls in both countries; schoolchildren from India do more physical activity than schoolchildren from Spain. Therefore, it is concluded that the level of physical activity is insufficient, decreases slightly with the age and is higher in India.
\end{abstract}

Keywords: Physical Activity, Health, Schoolchildren, Children, Spain, India. 


\section{Introducción}

La promoción de la práctica físico-deportiva se ha convertido en uno de los objetivos más importantes de los centros educativos de la mayoría de los países desarrollados, debido al elevado sedentarismo existente en los diferentes sectores de la población. Actualmente, la sociedad ha conferido al ejercicio físico y al deporte una función primordial en la preservación y desarrollo de la salud en el ser humano, de tal manera que son universalmente conocidas las aportaciones beneficiosas que una práctica de actividad físico-deportiva, realizada bajo unos determinados parámetros de frecuencia, intensidad y duración, provoca sobre la salud, situando la misma dentro de los modelos o estilos de vida saludables (Vílchez, 2007).

Sin embargo, los efectos positivos de la práctica de actividades físico-deportivas no se corresponden con la frecuencia de la práctica por parte de la población escolar. Al respecto, algunas investigaciones señalan un descenso significativo de la práctica físico-deportiva a medida que se va avanzando desde la infancia a la adolescencia (Moreno, Cervelló y Moreno, 2007; Román, Serra, Ribas, Pérez-Rodrigo y Aranceta, 2006; Perula de Torres, Lluch, Ruiz Moral, Espejo, Tapia y Mengual, 1998).

Esta contradicción ha provocado que la promoción de hábitos y estilos de vida saludables sea un objetivo prioritario de las sociedades desarrolladas. Resulta sorprendente que, a mayor índice de desarrollo sociocultural, peores son las condiciones de vida: mala alimentación, sedentarismo, adquisición de hábitos nocivos para la salud, entre otros (Perula de Torres et al., 1998).

Según las recomendaciones mundiales sobre la actividad física para la salud que establece la Organización Mundial de la Salud (OMS, 2010), para los niños y jóvenes de 5 a 17 años de edad, la actividad física consiste en juegos, deportes, desplazamientos, actividades recreativas, educación física o ejercicios programados, en el contexto de la familia, la escuela o las actividades comunitarias. Con el fin de mejorar las funciones cardiorrespiratorias y musculares y la salud ósea, así como reducir el riesgo de enfermedades no transmisibles (ENT), se recomienda que:

1. Los niños y jóvenes de 5 a 17 años inviertan como mínimo 60 minutos diarios en actividades físicas de intensidad moderada a vigorosa.

2. La actividad física por un tiempo superior a 60 minutos diarios reportará un beneficio aún mayor para la salud.

3. La actividad física diaria debería ser, en su mayor parte, aeróbica. Convendría incorporar, como mínimo tres veces por semana, actividades vigorosas que refuercen, en particular, los músculos y huesos. 
URL: http://www.revistas.una.ac.cr/mhsalud
Guillermo Felipe López Sánchez, Francisco

José Borrego Balsalobre, Laura López Sánchez y Arturo Díaz Suárez

Los últimos estudios sobre la actividad física realizada por los escolares españoles muestran que la gran mayoría de escolares de España (más del 65\%) realiza actividad física (Castells, Capdevila, Girbau y Rodríguez, 2006; García-Cantó, 2011; Hernández et al., 2007; Romero, Chinchilla y Jiménez, 2008; Vera, 2006). No obstante, también queda claro que la actividad física que realizan no suele satisfacer las recomendaciones de la OMS, como indican los estudios de Romero et al. (2008), Martínez-Gómez et al. (2009) o GarcíaCantó (2011). Asimismo, la mayoría de escolares indios realiza actividad física, pero -al igual que ocurre en España- no suelen satisfacer las recomendaciones de la OMS (Gulati et al., 2014). Por tanto, según los últimos estudios, los escolares de ambos países realizan actividad física, pero no la suficiente.

El objetivo de este artículo es analizar y comparar el nivel actual de actividad física habitual de escolares de 8-9 años de España e India, según edad, sexo y país, prestando especial atención al porcentaje de escolares activos e inactivos, así como al número medio de días en los que los escolares realizan la actividad física necesaria recomendada por la OMS.

\section{Metodología}

\section{Participantes}

En este estudio participaron 85 escolares de 8-9 años. Según la edad, la muestra se divide en 46 escolares de 8 años y 39 escolares de 9 años. Según el sexo, la muestra se compone de 41 niñas y 44 niños. Según la procedencia, 44 escolares son de España (región de Murcia) y 41 escolares proceden de India (distrito de Karbi Anglong, Assam). Todos los escolares participantes pertenecen a centros educativos públicos ubicados en zona urbana. El tipo de muestreo fue intencional. Todos los escolares participantes pertenecen a tres centros educativos públicos ubicados en zona urbana (un centro de España y dos centros de India). En los tres centros, los escolares recibían dos horas de Educación Física a la semana.

El estudio se realizó de acuerdo con la Declaración de Helsinki de 1961 (revisada en Tokyo en 1989 y en Edimburgo en 2000) y fue aprobado por el Comité de Ética de la Universidad de Murcia (España).

\section{Instrumentos}

El cuestionario empleado ha sido el PACE (Physician-based Assessmentand Counseling for Exercise), que valora con dos preguntas cuántos días en la última semana (PACE 1) y en una semana habitual (PACE 2) el sujeto realiza al menos 60 minutos de actividad física. Si el resultado compuesto obtenido en ambas preguntas ([PACE 1+PACE

Revista MHSalud® (ISSN: 1659-097X) Vol. 12. No. 2. Agosto-Febrero, 2016 
2]/2) es $\geq 5$ días, el sujeto es considerado activo (Prochaska et al., 2001; Martínez-Gómez et al., 2009). El cuestionario presenta una fiabilidad test-retest evaluada por el coeficiente de correlación intraclase (CCI) de 0,77. Aunque este cuestionario fue validado con adolescentes, resulta muy útil también en niños debido a su sencillez, fácil comprensión, rápida aplicación y a que las recomendaciones de actividad física de la OMS en las que se basa este cuestionario son las mismas para el grupo de edad de niños y jóvenes ( 5 a 17 años). El cuestionario fue validado inicialmente en inglés (Prochaska et al., 2001) y posteriormente validado en español (Martínez-Gómez et al., 2009). En India fue aplicado en inglés y en España, en español.

\section{Procedimiento}

Se realizó un estudio con un diseño cuantitativo no experimental, transversal y descriptivo, a través de encuestas para conocer el nivel de actividad física habitual de los escolares. Los cuestionarios fueron cumplimentados de forma anónima mediante auto-reporte. El personal investigador se encargó de contactar con las escuelas y distribuir los cuestionarios. Además, los participantes y sus padres/tutores declararon su consentimiento informado.

\section{Análisis estadístico}

Se ha realizado un análisis estadístico por medio del Statistical Package for Social Sciences 22 (SPSS-22) y se han seguido las indicaciones del Manual de Estadística Aplicada a las Ciencias de la Actividad Física y el Deporte (Ortega, Ortiz y Artés, 2009). Asimismo, se han puesto en práctica técnicas estadísticas de análisis descriptivo (frecuencias, porcentajes, media y desviación estándar) y se ha aplicado estadística inferencial (prueba $t$ de Student para muestras independientes) para determinar si existen diferencias significativas según edad, sexo y país de procedencia.

\section{Resultados}

En este apartado se presentan los resultados del estudio, prestando especial atención a los días por semana en los que la muestra realiza al menos 60 minutos de actividad física y a la clasificación de la población en activa e inactiva, teniendo en cuenta las diferencias según edad, sexo y país.

$\mathrm{Al}$ analizar las frecuencias y porcentajes de esta variable (días de la semana en los que la muestra realiza actividad física al menos 60 minutos), según la muestra total y según el país, se obtienen los resultados expuestos en la tabla 1. 
URL: http://www.revistas.una.ac.cr/mhsalud y Arturo Díaz Suárez

Tabla 1

Número de días a la semana en los que los estudiantes de la muestra realiza actividad fisica (al menos 60 minutos)

\begin{tabular}{ccccccc}
\hline \multirow{2}{*}{ Días/semana } & \multicolumn{2}{c}{ Muestra total/ $\mathrm{n}=85$} & \multicolumn{2}{c}{ España $^{*}$} & \multicolumn{2}{c}{ India* $^{* *}$} \\
\cline { 2 - 7 } & Frecuencia $_{(\mathrm{i})}$ & Porcentaje $_{(\mathrm{f} \%)}$ & Frecuencia & Porcentaje & Frecuencia $^{\text {Porcentaje }}$ \\
\cline { 2 - 7 } & 8 & 9.4 & 8 & 18.2 & 0 & 0 \\
2 & 10 & 11.8 & 7 & 15.9 & 3 & 7.3 \\
3 & 17 & 20.0 & 12 & 27.3 & 5 & 12.2 \\
4 & 14 & 16.5 & 7 & 15.9 & 7 & 17.1 \\
5 & 12 & 14.1 & 2 & 4.5 & 10 & 24.4 \\
6 & 17 & 20.0 & 7 & 15.9 & 10 & 24.4 \\
7 & 2 & 2.4 & 0 & 0 & 2 & 4.9 \\
\hline TOTALES & 5 & 5.9 & 1 & 2.3 & 4 & 9.8 \\
\hline
\end{tabular}

(*) Tamaño muestral España $n=44$

(**)Tamaño muestral India $\mathrm{n}=41$

Al analizar la media y la desviación estándar $(D E)$ de esta variable se obtienen los siguientes resultados (Tablas 2).

Tabla 2

Días a la semana en los que la muestra realiza actividad física al menos 60 minutos

\begin{tabular}{lllcl}
\hline \multirow{2}{*}{ Muestra total } & $N$ & MEDIA & DE \\
\cline { 3 - 5 } & & 85 & 3.13 & 1.91 \\
\hline \multirow{2}{*}{ Según Edad } & 8 años & 46 & 3.30 & 1.98 \\
& 9 años & 39 & 2.92 & 1.83 \\
\hline \multirow{2}{*}{ Según Sexo } & Niños & 41 & 3.66 & 1.91 \\
& Niñas & 44 & 2.64 & 1.79 \\
\hline \multirow{2}{*}{ Según País } & España & 44 & 2.32 & 1.79 \\
& India & 41 & 4.00 & 1.64 \\
\hline
\end{tabular}

El número medio de días por semana en los que la muestra del estudio realiza al menos los 60 minutos de actividad física diaria recomendada por la OMS es de 3,13 días, resultado inferior a los cinco días necesarios para ser considerada población activa, según el cuestionario PACE. 
Al analizar esta variable en función de la edad, se observa que los escolares de 8 años son ligeramente más activos que los de 9 años, realizando los de 8 años 3.3 días a la semana la actividad física recomendada frente a los 2.92 días los de 9 años.

En función del sexo, los niños realizan de media un día más a la semana de actividad física que las niñas; 3.66 días frente a los 2.64 días realizados por las niñas.

Si comparamos los datos de los dos países analizados en el estudio, se aprecia que los escolares de India realizan más actividad física que los de España: los indios 4 días por semana y los españoles 2.32 días, prácticamente la mitad.

Al aplicar estadística inferencial (prueba t de Student para muestras independientes) para determinar si hay diferencias significativas según edad, sexo y país de procedencia, se obtienen los siguientes resultados (Ver tabla 3):

Tabla 3

Valores reportados prueba t-student para muestras independientes

\begin{tabular}{llll}
\hline & t-student & $p$-vale & $n-1 \mathrm{gl}$ \\
\hline Según Edad & 0.978 & 0.331 & 84 \\
Según Sexo & 2.594 & 0.011 & 84 \\
Según País & -3.987 & 0.000 & 84 \\
\hline
\end{tabular}

(*)Según la edad, no existen diferencias significativas ( $p=0.331)$. Según el sexo, existen diferencias significativas $(p=0.011)$. Según el país, existen diferencias significativas $(p=0.000)$.

Considerando la variable de días necesarios para ser activo $(\geq 5$ días) e inactivo $(<5$ días), al analizar las frecuencias y porcentajes de esta variable se obtiene que, de acuerdo con la clasificación del cuestionario PACE, la muestra de este estudio es, en su mayoría, inactiva (77.6\%), frente al 22,4\% de escolares activos. En la Tabla 4 se presenta esta información.

Tabla 4

Clasificación de la muestra en función de

dias activos ( $\geq 5$ dias) e inactivos ( $<5$ días)

\begin{tabular}{lcc}
\hline & Frecuencia & Porcentaje \\
\hline Activos & 19 & 22.4 \\
Inactivos & 66 & 77.6 \\
\hline Total & 85 & 100 \\
\hline
\end{tabular}


En función de la edad, en la Tabla 5 se puede apreciar la existencia de más sujetos de 8 años activos (26.15) que de 9 años (17.9).

Tabla 5

Clasificación de la muestra en función de días activos ( $\geq 5$ dias) e inactivos ( $<5$ días) según edad

\begin{tabular}{clcc}
\hline & & Frecuencia & Porcentaje \\
\hline \multirow{3}{*}{8 años } & Activos & 12 & 26.1 \\
& Inactivos & 34 & 73.9 \\
\cline { 2 - 4 } & Total & 46 & 100 \\
\hline \multirow{4}{*}{9 años } & Activos & 7 & 17.9 \\
\cline { 2 - 4 } & Inactivos & 32 & 82.1 \\
\cline { 2 - 4 } & Total & 39 & 100 \\
\hline
\end{tabular}

En la Tabla 6 se presentan los datos según el sexo en el cual se aprecia que los niños son más activos que las niñas: $31.7 \%$ de niños activos frente a solo un $13.6 \%$ de niñas activas.

Tabla 6

Clasificación en función de dias activos

( $\geq 5$ días) e inactivos ( $<5$ dias) según sexo

\begin{tabular}{clcc}
\hline & & Frecuencia & Porcentaje \\
\hline \multirow{3}{*}{ Niños } & Activos & 13 & 31.7 \\
& Inactivos & 28 & 68.3 \\
\cline { 2 - 4 } & Total & 41 & 100 \\
\hline \multirow{3}{*}{ Niñas } & Activos & 6 & 13.6 \\
& Inactivos & 38 & 86.4 \\
\cline { 2 - 4 } & Total & 44 & 100 \\
\hline
\end{tabular}


Finalmente, la tabla 7 expone datos en función del país en la cual se evidencia que el porcentaje de escolares activos en España (18.2 \%) es menor que el porcentaje de escolares activos en India (26.8 \%).

\section{Tabla 7}

Clasificación en función de días activos ( $\geq 5$ días) e inac-

tivos $(<5$ dias) según país

\begin{tabular}{clcc}
\cline { 3 - 4 } & & Frecuencia & $2.2733 \mathrm{~cm}$ \\
\hline \multirow{3}{*}{ España } & Activos & 8 & 18.2 \\
& Inactivos & 36 & 81.8 \\
\cline { 2 - 4 } & Total & 44 & 100 \\
\hline \multirow{3}{*}{ India } & Activos & 11 & 26.8 \\
& Inactivos & 30 & 73.2 \\
\cline { 2 - 4 } & Total & 41 & 100 \\
\hline
\end{tabular}

\section{Discusión}

Los resultados de este estudio se pueden comparar con los de otras investigaciones que se han centrado en el análisis del nivel habitual de actividad física en edad escolar. En primer lugar, se discuten los resultados de este estudio en relación con investigaciones que han empleado el mismo cuestionario (PACE) y, posteriormente, se discuten respecto a estudios que han empleado otros cuestionarios.

Prochaska et al. (2001) aplicaron el cuestionario PACE a una muestra de 250 sujetos (140 chicas y 110 chicos) de San Diego (California) y Pittsburgh (Pensilvania), con una media de edad de 14.6 años ( $\mathrm{DE}=1.4$ años), y encontraron los siguientes resultados: los sujetos de la muestra realizaban actividad física (60 minutos o más) una media de 2,4 días a la semana $(\mathrm{DE}=1.9)$. Los resultados de Prochaska et al. (2001) son casi idénticos a los resultados de este estudio en los escolares españoles, los cuales realizan actividad física (60 minutos o más) una media de 2.32 días a la semana $(\mathrm{DE}=1.79)$. En cambio, son inferiores a los niveles de actividad física de los escolares de India, los cuales realizan actividad física cuatro días/semana ( $\mathrm{DE}=1.64)$.

Martínez-Gómez et al. (2009) aplicaron el cuestionario PACE a una muestra de 200 adolescentes (99 chicos y 101 chicas) de la Comunidad de Madrid (España), con edades comprendidas entre los 13 y 17 años, y encontraron los siguientes resultados: los chicos realizaban actividad física (60 minutos o más) una media de 3.42 días a la semana ( $\mathrm{DE}=1.52$ ), mientras que las chicas realizaban una media de 2.48 días a la semana $(\mathrm{DE}=1.42)$. Los resultados de esta investigación son similares a los del presente estudio, en el que los niños realizan actividad física 3,66 días/semana ( $\mathrm{DE}=1.91$ ), mientras que las niñas solamente 2.64 días/semana ( $\mathrm{DE}=1.79)$. 
En cuanto a la comparación de este estudio con investigaciones que usaron otros cuestionarios, Castells et al. (2006), en una muestra de 2.400 escolares de 11 a 13 años de la provincia de Barcelona, observan que el $83 \%$ de los sujetos afirman realizar algún tipo de práctica físico-deportiva. Estos resultados contrastan con los de la presente investigación en la que un $77.6 \%$ de la muestra queda clasificada como inactiva; no obstante, es difícil comparar los resultados debido a que no se ha clasificado a los sujetos siguiendo el mismo protocolo.

Vera (2006), con una muestra de 1087 alumnos de 10 y 11 años de la Región de Murcia, señala que el $86.5 \%$ de los escolares practica actividad físico-deportiva alguna vez por semana. En la misma línea, García-Cantó (2011), en un estudio realizado con 1.200 escolares de 10 a 12 años de la región de Murcia, señala que el $79.4 \%$ de los escolares practica actividad físico-deportiva fuera del colegio. De nuevo, estos resultados contrastan con el elevado porcentaje de sujetos inactivos encontrados en el presente estudio (77.6\%), aunque se debe tener en cuenta que para ser considerados activos en este, se exigía realizar actividad física al menos 60 minutos diarios durante cinco días o más por semana, de acuerdo con las recomendaciones de la OMS.

Romero, Chinchilla y Jiménez (2008), en un estudio con alumnos de 12 años de edad de la provincia de Málaga, muestran que únicamente el $14 \%$ de los alumnos realiza actividad física diaria en su tiempo libre. Los resultados de Romero et al. se asemejan más a los del presente estudio, sobre todo a los de la muestra española, en la que solo el $18.2 \%$ es considerado activo. Difieren algo más con la muestra de India, en la que el $26.8 \%$ de los escolares es activo.

Hernández et al. (2007), en una investigación con 2.834 escolares de 10 a 17 años de seis ciudades de diferentes Comunidades Autónomas, destacan que un alto porcentaje de escolares $(66.2 \%)$ realiza actividad físico-deportiva fuera del entorno escolar más de dos días a la semana. Estos resultados contrastan con los del presente estudio, en el que un 38.6\% de los escolares españoles realizan al menos 60 minutos de actividad física más de dos días a la semana, frente al $80.5 \%$ de escolares indios que practican al menos 60 minutos de actividad física más de dos días a la semana.

Por último, García (2011), en un estudio realizado con 1.200 escolares de 10 a 12 años de la región de Murcia, señala que un 36.7 \% de los escolares realiza actividades físico-deportivas tres o más días a la semana. Estos resultados son muy similares a los encontrados en la muestra española de este estudio, en el que el $38.6 \%$ de los escolares españoles realiza al menos 60 minutos de actividad física tres o más días a la semana, pero difieren del $80.5 \%$ de escolares indios que practican al menos 60 minutos de actividad física tres o más días a la semana. 


\section{Conclusiones y recomendaciones}

La muestra estudiada no realiza suficiente actividad física, según las recomendaciones de la OMS. Casi el $80 \%$ de la muestra es inactiva según la clasificación del cuestionario PACE. En función de la edad, los escolares de 8 años son ligeramente más activos que los de 9 años, aunque no existen diferencias significativas. Según el sexo, los niños realizan más actividad física que las niñas, existiendo diferencias significativas. Asimismo, en función del país, los escolares de India realizan más actividad física que los de España, existiendo también diferencias significativas.

Se debe aumentar la práctica de actividad física en ambos países y sobre todo en España donde el problema es mucho mayor. Una manera de aumentar la práctica de actividad física habitual en escolares sería abordar intervenciones multidisciplinares que refuercen los hábitos de práctica físico-deportiva de los escolares. Desde el ámbito de la Educación Física y, apoyada desde las entidades locales, se deberían implementar programas de práctica físico-deportiva que despierten el interés por parte de los escolares, sobre todo del sexo femenino.

Es conveniente la elección de un cuestionario de referencia que permita una comparación sencilla y directa entre diferentes muestras. El uso de diferentes cuestionarios genera confusión y dificulta la discusión entre investigaciones.

Como futuras líneas de investigación, se recomienda llevar a cabo investigaciones similares, profundizando en el análisis estadístico de las variables centrales, para entender en mayor medida las relaciones e interacción entre las mismas, de tal forma que los investigadores dispongan de un mayor conocimiento acerca del nivel de actividad física habitual de los escolares de diferentes países del mundo.

\section{Referencias bibliográficas}

Castells, M., Capdevila, C., Girbau, T. y Rodríguez, C. (2006). Estudio del comportamiento alimentario en escolares de 11 a 13 años de Barcelona. Nutrición hospitalaria, 21 (4), 517-532.

García-Cantó, E. (2011). Niveles de actividad física habitual en escolares de 10 a 12 años de la Región de Murcia. Tesis doctoral. Murcia: Universidad de Murcia.

Gulati, A., Hochdorn, A., Paramesh, H., Paramesh, E. C., Chiffi, D., Kumar, M.,...Baldi, I. (2014). Physical Activity Patterns Among School Children in India. Indian Journal of Pediatrics, 81 (Suppl 1), S47-S54. http://dx.doi.org/10.1007/s12098-014-1472-X 
Hernández, J. L., Velázquez, R., Alonso, D., Garoz, I., López, C., López, A., Maldonado, A., Martínez, M. E., Moya, J. M. y Castejon, F. J. (2007). Evaluación de ámbitos de la capacidad biológica y de hábitos de práctica de actividad física. Estudio de la población escolar española. Revista de Educación, 343, 177-198. Recuperado de: http://www. revistaeducacion.mec.es/re343/re343 09.pdf

Martínez-Gómez, D., Martínez-De-Haro, V., Del-Campo, J., Zapatera, B., Welk, G. J., Villagra, A., Marcosa, A. y Veiga, O. L. (2009). Validez de cuatro cuestionarios para valorar la actividad física en adolescentes españoles. Gaceta Sanitaria, 23 (6), 512-517. http://dx.doi. org/10.1016/j.gaceta.2009.02.013

Moreno, J. A., Cervelló, E. y Moreno, R. (2007). El autoconcepto físico como predictor de la intención de ser físicamente activo. Psicología y salud, 17(2), 261-267.

OMS (2010). Recomendaciones mundiales sobre actividad fisica para la salud. Suiza: Organización Mundial de la Salud.

Ortega, E., Ortiz, I. M. y Artés, E. M. (2009). Manual de Estadística Aplicada a las Ciencias de la Actividad Física y el Deporte. Murcia: Diego Marín.

Perula de Torres, L. A., Lluch, C., Ruiz Moral, R., Espejo, J., Tapia, G. y Mengual, P. (1998). Prevalencia de actividad física y su relación con variables sociodemográficas y ciertos estilos de vida en escolares cordobeses. Revista española de salud pública, 72 (3), 233-244.

Prochaska, J. J., Sallis, J. F. y Long, B. (2001). A physical activity screening measure for use with adolescents in primary care. Archives of Pediatrics \& Adolescent Medicine, 155 (5), 554-559. http://dx.doi.org/10.1001/archpedi.155.5.554

Román, B., Serra, L., Ribas, L., Pérez-Rodrigo, C. y Aranceta, J. (2006). Actividad física en la población infantil y juvenil española en el tiempo libre. Estudio Enkid (1998-2000). Apunts. Medicina de l'esport, 41 (151), 86-94.Recuperado de: http://www.apunts.org/es/ actividad-fisica-poblacion-infantil-juvenil/articulo/13096378/

Romero, O., Chinchilla, J. L. y Jiménez, A. (2008). Utilización del tiempo libre, hábitos de alimentación y condición física de los escolares de doce años de edad, según variables sociodemográficas. Revista Fuentes (8). Recuperado de: http://www.quadernsdigitals.net/ datos/hemeroteca/r_36/nr 799/a 10787/10787.pdf

Vera, J.A. (2006). Evaluación participativa y responsabilidad en Educación Física. Tesis doctoral. Murcia: Universidad de Murcia. 
Vílchez, G. (2007). Adquisición y mantenimiento de hábitos de vida saludables en los escolares de tercer ciclo de Educación Primaria de la Comarca granadina de los Montes Orientales y la influencia de la Educación física sobre ellos. Tesis Doctoral. Granada: Universidad de Granada. Recuperdado de : http://hera.ugr.es/tesisugr/16729158.pdf

Recepción: 21 de abril del 2015

Corrección: 20 de enero del 2016.

Aceptación: 25 de enero del 2016.

Publicación: 29 de enero del 2016.

1 Guillermo Felipe López Sánchez. Licenciado en Ciencias de la Actividad Física y del Deporte. Maestro (especialidad Educación Física). Máster Universitario en Investigación en Educación Física y Salud. Máster Universitario en Formación del Profesorado de Educación Secundaria Obligatoria y Bachillerato, Formación Profesional y Enseñanza de Idiomas (especialidad de Educación Física). Investigador del Grupo de investigación deporte, gestión y recreación (INGESPORT). Profesor de la Facultad de Ciencias del Deporte, Universidad de Murcia, España.

MD. Dilsad Ahmed. Licenciatura en Educación Física. Máster en Educación Física. Máster en Filosofía (especialidad Psicología del Deporte). Doctor en Filosofía (especialidad Educación Física). Investigador ayudante en la Facultad de Educación, Universidad de Macau, República Popular de China.

Francisco José Borrego Balsalobre. Licenciado en Ciencias de la Actividad Física y el Deporte. Maestro (especialidad Educación Física). Máster en Gestión y Planificación de Entidades Deportivas. Máster Universitario en Investigación en Educación Física y Salud. Doctor en Psicología de la Actividad Física y el Deporte. Investigador del Grupo de investigación deporte, gestión y recreación (INGESPORT) de la Universidad de Murcia (España).

Laura López Sánchez. Licenciada en Ciencias de la Actividad Física y del Deporte. Maestra (especialidad Educación Física). Máster de investigación en Investigación en Ciencias de la Actividad Física y el Deporte. Investigadora del Grupo de investigación deporte, gestión y recreación (INGESPORT) de la Universidad de Murcia (España).

Arturo Díaz Suárez. Licenciado en Educación Física. Doctor en Ciencias de la Educación. Profesor titular y decano de la Facultad de Ciencias del Deporte de la Universidad de Murcia. Investigador principal del Grupo de investigación deporte, gestión y recreación (INGESPORT) de la Universidad de Murcia (España). 